\title{
Selective Bit-error Checking at the MAC Layer for Voice Over Mobile Ad Hoc Networks with IEEE 802.11
}

\author{
H. Dong, I. D. Chakares, A. Gersho, E. Belding-Royer ${ }^{\dagger}$, J. D. Gibson \\ Department of Electrical and Computer Engineering \\ Department of Computer Science ${ }^{\dagger}$ \\ University of California, Santa Barbara, CA 93106 \\ Email: \{huidong, gersho,gibson\}@ece.ucsb.edu, idc@engineering.ucsb.edu, ebelding@cs.ucsb.edu
}

\begin{abstract}
Mobile ad hoc networks (MANET) have more severe operating conditions than traditional wireless networks. The MAC protocol of IEEE 802.11 mitigates collisions and ensures error-free packet transmissions at the cost of limiting capacity and increasing latency. For voice transmission over MANETs this cost should be minimized. We propose and examine selective error checking (SEC) at the MAC layer of 802.11 that takes advantage of the fact that many of the speech bits can tolerate errors while other bits must be protected for effective reconstruction of the speech. Simulation results demonstrate that the network performance and the speech quality are substantially improved by modifying the MAC layer with SEC to suit a particular GSM speech compression standard, the Narrow-Band Adaptive MultiRate (NB-AMR) coder operating at a rate of 7.95 kbps. ${ }^{1}$
\end{abstract}

\section{INTRODUCTION}

A mobile ad hoc network (MANET) is a wireless LAN (WLAN) wherein mobile nodes can communicate with one other without relying on any pre-existing infrastructure. In a MANET, all the mobile nodes have equal capabilities and operate not only as hosts but also as network routers. Communication links might be broken and a dynamic routing protocol is needed. Dynamic routing requires a nontrivial amount of overhead traffic on the network to discover changes in the available paths or to identify on demand a specific route when needed. The efficiency of the routing protocol and other higher layer protocols such as IP can indirectly depend on the underlying medium access control (MAC) protocol.

The IEEE 802.11 [1], includes a MAC protocol and a physical (PHY) layer protocol. The MAC protocol mitigates collisions and ensures error-free packet transmissions at the cost of limiting capacity and increasing latency. Many research efforts have concentrated on improving the performance and capacity of the 802.11 MAC protocol for data. Also, some studies have focused on modifying the IEEE 802.11 MAC protocol to support real-time traffic, such as voice, in MANETs. In general, the performance of real-time communication in MANETs can be improved by reserving bandwidth and giving

\footnotetext{
${ }^{1}$ This work is supported in part by the NSF under grants EIA-9986057, EIA0080134 and CCR-0243332, the University of California MICRO Program, Dolby Laboratories, Lucent Technologies, Microsoft, and Qualcomm.
}

higher priority to real-time data traffic. However, none of these schemes has considered the varying perceptual sensitivity of different bits in a speech frame.

The CRC mechanism in the 802.11 MAC protocol is used for the entire packet (the MAC frame) to make sure the received packet is error-free. This may lead to multiple retransmissions and possibly dropping a packet. However, this is not efficient for voice transmission which is delay-sensitive and relatively tolerant to bit errors. The number of dropped packets is decreased and bandwidth is conserved if some bit errors are allowed in voice data.

This paper introduces selective error checking (SEC) at the MAC layer to accept non-critical bit errors in voice data so that retransmission and dropping of MAC packets are reduced. The effectiveness of this scheme is examined by measuring the network performance in terms of packet loss and average end-to-end packet transmission delay, and by assessing the subjective quality of the received speech. The results clearly demonstrate the performance advantage of our proposed scheme.

The paper is outlined as follows. In Section II, the IEEE 802.11 MAC protocol is overviewed and some related work is reported. In Section III, the problem of voice transmission over MANET is discussed and relevant speech signal processing techniques are reviewed. The SEC modified IEEE 802.11 MAC protocol is proposed here. Section IV verifies the proposed MAC protocol experimentally in terms of the network performance and speech quality. Conclusions are presented in Section V.

\section{IEEE 802.11 MAC PROTOCOL}

\section{A. Standard Overview}

The original IEEE 802.11 standard defines a MAC protocol, and three alternative physical (PHY) layers. The IEEE 802.11 standard has been updated for higher rate extensions; however, a common MAC protocol is widely used to interact with the various physical layers.

The IEEE 802.11 MAC layer defines two different access methods. Only one of these, the distributed coordination function (DCF), is applicable to MANET. This method is 
basically carrier sense multiple access with collision avoidance (CSMA/CA). DCF offers two techniques for transmission of a MAC frame: the basic access scheme and the Request-toSend/Clear-to-Send (RTS/CTS) access scheme.

The basic access scheme is a two-way handshaking technique. Before a node initiates a transmission, it senses the channel to determine whether another node is transmitting. If the medium is busy, the transmission is deferred until the end of the ongoing transmission. If the medium is found to be idle for an interval, the node starts its transmission. A positive acknowledgment (ACK) is employed after a 32-bit cyclic redundancy code (CRC) is computed over the received packet and the entire packet is error-free. If an ACK packet is not received, the data packet is presumed to have been lost, and a retransmission is scheduled. After 7 failed attempts for small packets or 4 for large packets, the data packet is dropped.

The RTS/CTS access scheme is an extension of the basic access scheme. A RTS/CTS exchange is performed before transmission of a data packet. This is helpful for reducing collisions due to hidden nodes, but the additional overhead is justified only for very large data packets. Therefore, the RTS/CTS access scheme is not used for voice transmission.

\section{B. Related Work}

Since the IEEE 802.11 was standardized, there have been many research efforts conducted on analyzing and improving the performance and capacity of the 802.11 MAC protocol for WLANs and supporting real-time traffic [2]-[6].

The most efficient way to transmit voice data is to employ a reservation scheme that guarantees delay and bandwidth. Many different reservation schemes have been studied in [7][11]. Moreover, a new standard, IEEE 802.11e, is under development to support delay-sensitive applications for Quality of Service (QoS) with multiple managed levels of QoS for data, voice, and video applications.

A retransmission scheme was also investigated to give distinct treatment to each of two classes of speech [12]. The packets are classified into two types, voiced and unvoiced, and the more critical voiced packets are protected by using a higher retry limit in the case of high packet loss rates.

During the research on voice over MANETs conducted in the Signal Compression Lab and Mobility Management and Networking Lab at UCSB, we examined the IEEE 802.11 MAC protocol and proposed the idea of allowing bit errors in the voice by modifying the MAC layer. Some improvement in network performance for three single-hop WLAN scenarios was reported in [13] where error checking of the voice data was fully omitted.

\section{Voice Transmission Over Ad Hoc Networks}

\section{A. Network Model}

A simple MANET with three colinear nodes is shown in Fig. 1. Nodes $\mathrm{A}$ and $\mathrm{C}$ are out of range for either direction of transmission (e.g., they cannot hear each other, while A and $\mathrm{B}$ and also $\mathrm{B}$ and $\mathrm{C}$ can hear each other). For voice communication between nodes $\mathrm{A}$ and $\mathrm{C}$, an intermediate node is needed to forward the packet and B serves this role here. Let $\mathrm{A}$ be the source node where the speech signal is generated, compressed and packetized with the UDP/IP protocol stack. The MAC frame in this case consists of the voice data, the UDP header, the IP header and the MAC header as shown in Fig. 1.

We assume for simplicity here that the nodes have a fixed location for the duration of a voice call, so they are not currently mobile. However, since the nodes are capable of mobility, a routing protocol, an essential part of any MANET, operates at node A to discover node B as the desired nextnode. Each successive packet is transmitted over the wireless medium by the 802.11 physical layer. At node $\mathrm{B}$, the routing protocol and the 802.11 protocol are involved to identify $\mathrm{C}$ as the next-node (and final destination) and deliver the packet to $\mathrm{C}$. At the destination node $\mathrm{C}$, the received packets are buffered, reordered as needed, unpacketized, and the voice data is uncompressed and speech is reconstructed.

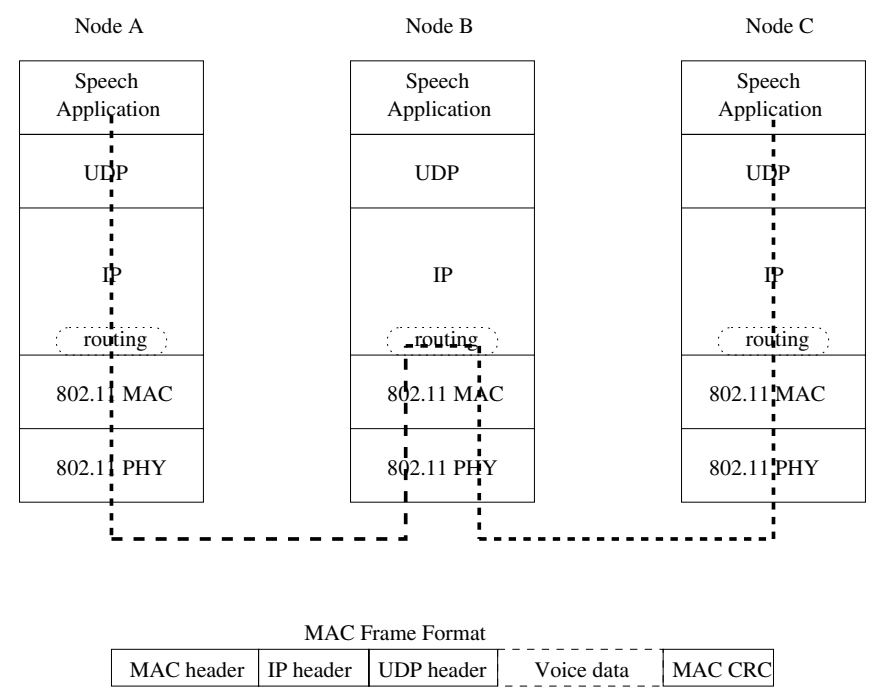

Fig. 1. A simple voice transmission model over a MANET

In our simple 3 node network, a collision occurs either when node $\mathrm{A}$ and $\mathrm{C}$ are sending frames to node $\mathrm{B}$ during the same time slot, or when node A and B (or B and C) are each attempting to send frames at same time. The bit errors in this frame, on the other hand, are caused by noise on the wireless link. The more bits in the frame, the higher the probability that the frame will have bit errors causing the CRC check to fail. Thus, smaller frames are preferred for unreliable channels. The size of the headers are the same for all frames, so the size of a frame varies with the size of a voice packet. The voice duration in a packet is always limited by to the applicationspecific delay requirements.

\section{B. Speech Coding}

Over the years, highly effective speech compression algorithms have been developed with increasing sophistication. Most codecs in use today are narrow-band speech coders that operate on speech that has been limited to the traditional 
telephone bandwidth of $3.5 \mathrm{KHz}$ and sampled at 8,000 samples per second. The input speech is partitioned into frames of 10 to $30 \mathrm{~ms}$ and for each frame computation is performed to extract a number of parameters that allow the decoder to synthesize a close approximation to this segment of speech. Usually in VoIP, only one frame is contained in each packet to avoid extra latency. A bit error in one of the more significant bits of a parameter such as pitch (typically 7 or 8 bits) can audibly degrade the reconstructed speech.

A large number of speech coders have been standardized for various applications. Generally, for wired VoIP applications and telephone bandwidth input speech, the ITU G.711 standard at $64 \mathrm{kbps}$ (essentially uncompressed raw speech), is used when the relative traffic load is expected to be low. For higher traffic wired VoIP applications, G.729 at $8 \mathrm{kbps}$ is widely used with very good speech quality. There is also an extensive set of speech coding standards for digital cellular applications worldwide such as the Narrowband Adaptive Multi-Rate (NBAMR) speech coder standardized by ETSI in 1998 [14].

The NB-AMR codec operates on speech frames of 20 ms (160 samples) each and is capable of encoding speech at 8 different bit rates, one of which is $7.95 \mathrm{kbps}$, chosen for our simulations. The NB-AMR speech coder delivers the similar speech quality to G.729 speech coder used in wired VoIP applications and the rate of $7.95 \mathrm{kbps}$ is nearly equal to that of G.729. The codec also has optional features that include silence compression, voice activity detection, forward error correction, and error concealment techniques. Most of these features have specifically been designed for wireless applications.

\section{Error Concealment}

Error concealment algorithms have been extensively studied to recover a lost voice packet and to conceal bit errors in a received voice packet in voice over IP and digital cellular applications. Generally, the short-term self-similarity feature of speech signals is used to produce a replacement for a lost packet and to conceal the effect of bit errors in a received packet. The techniques based on insertion, interpolation and regeneration of a speech waveform are usually utilized. Error concealment is necessarily codec-dependent and NB-AMR employs this technique effectively.

\section{Selective Error Checking}

The bits in an NB-AMR voice frame are not of equal perceptual importance, and can be classified according to their sensitivity. For example, there are 159 bits in a speech frame coded by NB-AMR at $7.95 \mathrm{kbps}$, where 75 bits are classified as class A that is important and 84 bits are classified as class B that is relatively unimportant. Errors in class A may not be acceptable while errors in class B can be tolerated or concealed. This non-uniform bit sensitivity has been exploited for cellular applications to design forward error correction channel coding schemes that deliver different levels of protection to different classes of bits in digital cellular applications. Until now, this feature has not been utilized in IEEE 802.11-based WLANs.
We propose here an SEC scheme in the MAC layer to take advantage of the already known non-uniform bit sensitivity of the NB-AMR speech signal. The CRC in the MAC layer checks the bits in all headers and the most important bits of the voice data. Compared with the original CRC checking scheme that operates over an entire packet, SEC decreases the number of MAC frame retransmissions and dropped frames at each link, thereby reducing latency and traffic loading on the network.

In conjunction with SEC at the MAC layer, it is necessary to disable the UDP checksum (by setting the checksum to zero). Otherwise, packets arriving with errors in the low sensitivity portion of the voice data will be dropped even though these errors have been ignored at the MAC layer. Note that the UDP checksum is an optional operation and can be disabled without any modification of the transport layer.

\section{E. Performance Evaluation}

To examine the effectiveness of the SEC voice over MANETs, we evaluate the system performance by the following measurements:

Average end-to-end packet transmission delay is the difference between the packet transmission time at the source and its reception time at the destination. The delay requirement varies with different applications. A two-way conversation delay, including the end-to-end packet transmission delay, the coding delay and the buffer delay etc., may range from $80 \mathrm{~ms}$ to $400 \mathrm{~ms}$, while one-way streaming can have longer latency. A packet may be dropped if its delay exceeds the maximum allowed delay. In our research, we are interested in the delay improvement by using the new MAC protocol, so packets with long delay are not dropped.

Packet loss rate is the ratio of non-received-packets at the destination and the sent-packets at the source. The packets are lost in MAC layer for the following reasons: (i) when the medium is busy, packets are queued. If the packet buffer overflows, packets are dropped; (ii) when a packet collides or the MAC layer CRC fails, retransmission of the packet is required. If the number of unsuccessful retransmissions reaches the maximum, the packet is dropped.

Subjective speech quality is evaluated by an informal listening test. A group of ten untrained listeners are asked to give their preferences in a series of A-B comparison tests.

Jitter is another important performance metric. It is the variance in inter-arrival time between consecutive packets reaching the destination. Speech quality can be very different for different jitter characteristics. Generally jitter can be traded off for additional delay by buffering packets before further processing at the destination. In this paper we assume an ideal buffer used at the destination, and do not consider jitter.

\section{Simulations AND Results}

\section{A. Network Scenario}

A network scenario shown in Fig. 2 is examined by NS2 simulator [15] in this research. The distances between the nodes are chosen so that the nodes are at a "hearing" distance 


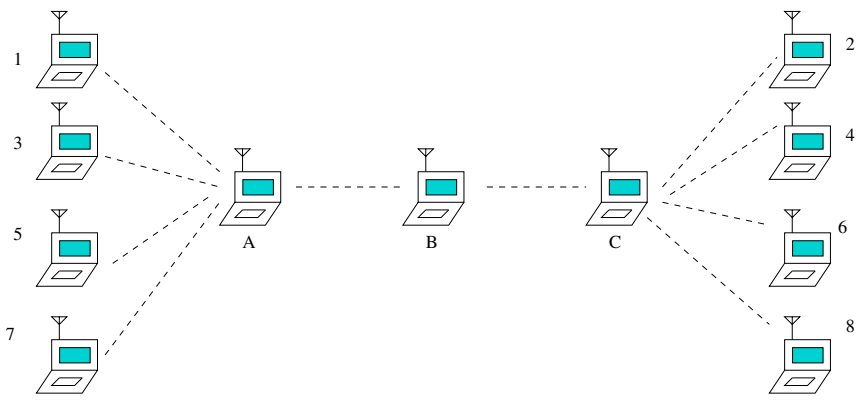

Fig. 2. Network Scenario

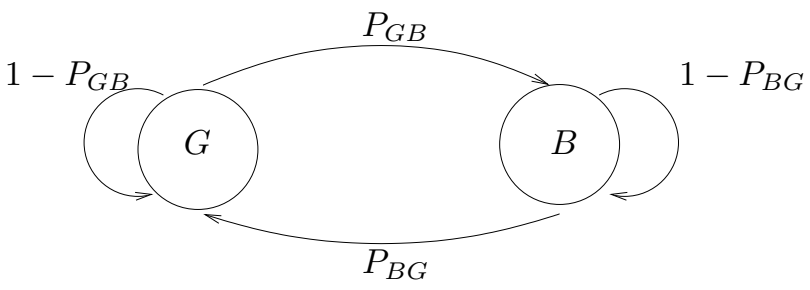

Fig. 3. Elliott-Gilbert Error Model

from each other and share the medium. The data rate of IEEE 802.11 was configured to be 2 Mbps.

In our simulation model, the nodes have no mobility. This is primarily because our interest in this paper is to focus on the effectiveness of SEC at the MAC layer. Hence, we assume that the network is fixed for the duration of the voice transmission, yet the normal overhead traffic due to dynamic routing protocol is present. Specifically, we use the destination sequenced distance vector (DSDV) routing protocol [16].

To evaluate the effectiveness of the new MAC protocol for multihop transmission, one voice transmission is separately simulated from node 1 to $\mathrm{A}, 1$ to $\mathrm{B}, 1$ to $\mathrm{C}$, and 1 to 2 .

To evaluate the effectiveness of the new MAC protocol for multiple traffic transmission, 1, 2, 3, and 4 voice transmissions from node 1 to node B are separately simulated.

The Elliott-Gilbert two state Markov model [17], [18], shown in Fig. 3, is used to model the wireless channel. The bit errors generated by this model are introduced to MAC frames. In this model, each state represents a binary symmetric channel. Bit errors occur with low probability $p_{G}$ in the "good" state $(G)$, and bit errors happen with high probability $p_{B}$ in the "bad" state $(B) . p_{G B}$ and $p_{B G}$ represent the probability of switching from the good state to the bad state and vice versa. An average link proposed in [19] is used in our simulation where $p_{G}=0, p_{B}=0.33, p_{G B}=0.01$ and $p_{B G}=0.14$. Based on the Elliott-Gilbert model, this results in an average error probability for this link of 0.022 .

\section{B. Voice Transmission}

For our simulation, we use 25 seconds of speech, consisting of 4 male sentences and 4 female sentences modeled as a constant bit rate traffic source with $20 \mathrm{~ms}$ of speech for each packet. After the speech is compressed by the NB-AMR coder at $7.95 \mathrm{kbps}$, there are 159 bits (20 bytes) for a $20 \mathrm{~ms}$ voice data packet where 75 bits are classified as class A and 84 bits are classified as class B. A MAC frame contains 28 bytes of MAC header, 20 bytes of IP header, 8 bytes of UDP header and 20 bytes of voice data. That is, a 76-byte MAC frame is sent from the source every $20 \mathrm{~ms}$, and the voice transmission rate is $7.95 \mathrm{kbps}$.

For each transmission listed in Section IV-A, both the original IEEE 802.11 MAC protocol and a modified IEEE 802.11 MAC protocol are tested separately. The two MAC protocols are differentiated by the type of CRC error checking mechanism.

During the transmission with the original MAC protocol, for each hop the CRC at the MAC layer checks the MAC header, the UDP/IP header and the entire voice data. If there is any bit error in the entire 76 byte data, a MAC layer retransmission is needed, or this MAC frame is discarded if the number of unsuccessful retransmissions has reached the maximum. At the network layer of the receiver, the CRC in the IP header protects the IP header. At the transport layer, the CRC in the UDP header protects the UDP header, the IP pseudoheader and the voice data.

During the transmission with the modified MAC protocol, the data is transmitted in a similar way. But, for each hop, the $\mathrm{CRC}$ at the MAC layer ascertains 66 byte data error-free that contains all headers and the class A voice data. The CRC in the UDP protocol is disabled to allow some errors in the voice data. It is simply done by setting the UDP checksum bits to zero in packets.

\section{Performance Analysis}

The packet loss rate and the average end-to-end packet transmission delay are measured to evaluate the effectiveness of the modified MAC protocol, that is, the effectiveness of SEC at the MAC layer. The results of one voice transmission over 1, 2, 3 and 4 hops are shown in Figs. 4 and 5, and the results of $1,2,3$, and 4 voice transmission over 2 hops are shown in Figs. 6 and 7. As we see, the network performance under severe channel conditions is notably improved by using the modified MAC protocol.

The network performance is improved because we allow some bit errors in voice data to increase the capacity and to decrease the latency. In the case of one-hop one-transmission, using the original MAC protocol and the modified MAC protocol, the packet loss rate and the average end-to-end packet transmission delay are small; the improvement by using SEC is not significant in our simulation, shown in Figs. 4 and 5. However, in the case of the two-hop one-transmission in our simulation, the packet loss rate is decreased from $18 \%$ to $10 \%$ by using the modified MAC protocol, but about $6 \%$ of the received packets are erroneous. Speech signal processing techniques are applied to conceal these errors and to recover the lost packets.

An informal listening test was conducted to evaluate the quality of speech from the two-hop one-transmission simulation. Ten people were asked to give their preference by listening to two pairs of samples shown in Table I. The results 


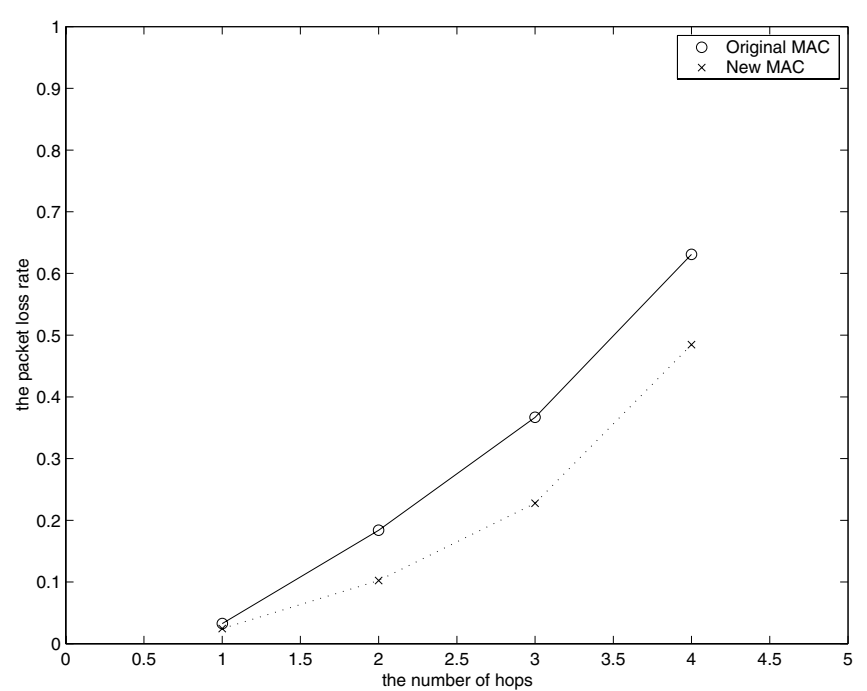

Fig. 4. Packet loss rate vs. number of hops

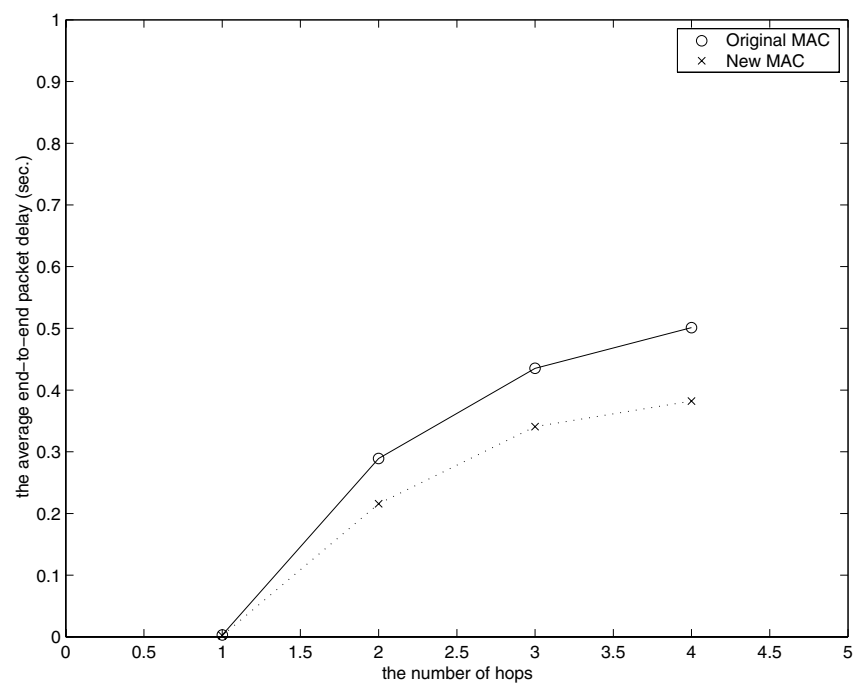

Fig. 5. Average end-to-end packet transmission delay vs. number of hops

show that nine out of ten listeners prefer the speech from the modified MAC protocol if no error concealment algorithm is applied and all ten listeners prefer the speech from the modified MAC protocol after the error concealment algorithm is employed.

\section{Conclusions}

In this paper, we proposed the SEC mechanism at the IEEE 802.11 MAC layer for voice over MANETs. We have shown that SEC improves the network performance and enhances the speech quality. While further research is needed to make voice over MANETs practical, it is evident that SEC can be combined with many other schemes for voice over MANETs, such as reservation schemes or high priority schemes, header compression, error concealment and silence compression. SEC is simple to implement and it is effective in conserving bandwidth and reducing latency for voice communication over

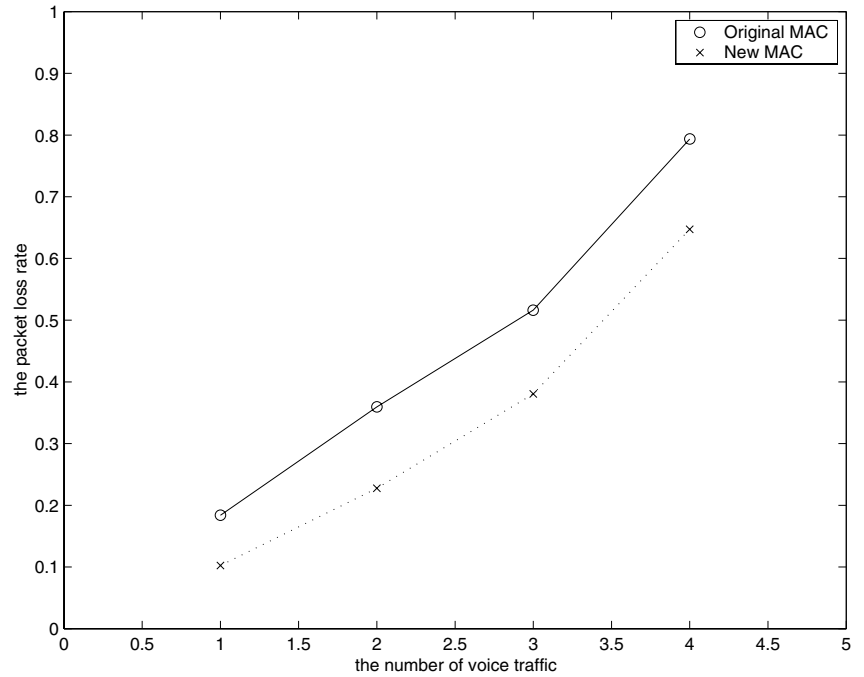

Fig. 6. Packet loss rate vs. number of voice traffic

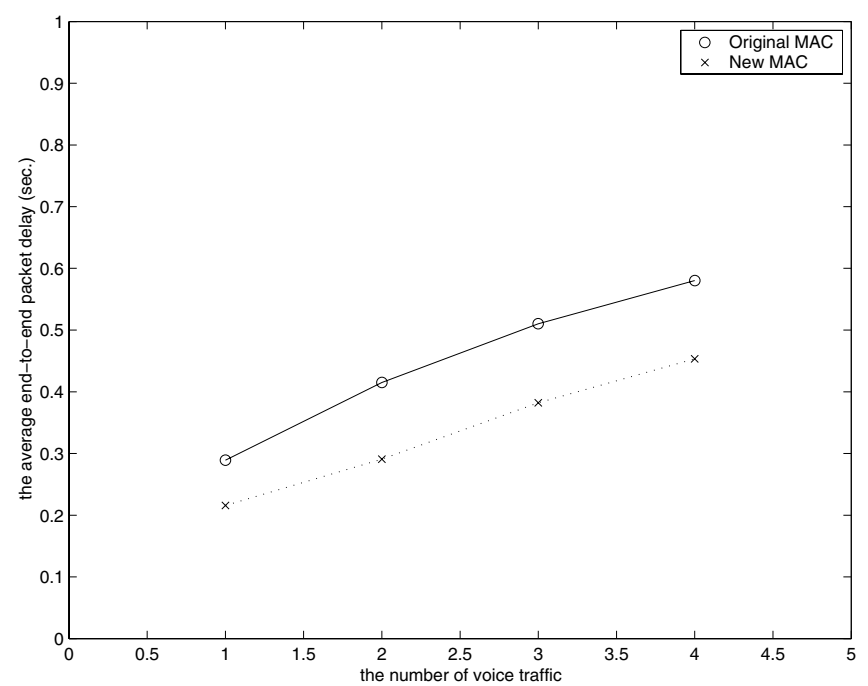

Fig. 7. Average end-to-end packet transmission delay vs. number of voice traffic

MANETs.

\section{REFERENCES}

[1] ISO/IEC 8802-11, ANSI/IEEE Std.802.11, Wireless LAN Medium Access Control (MAC) and Physical Layer (PHY) Specifications, 1999.

[2] F. Eshghi and A. K. Elhakeem, "Performance analysis of ad hoc wireless LANs for real-time traffic," in IEEE Journal on Selected areas in communications, Feb. 2003, vol. 21, pp. 203-122.

[3] O. Tickoo and B. Sikdar, "On the impact of IEEE $802 \mathrm{MAC}$ on traffic characteristics," in IEEE Journal on Selected areas in communications, Feb. 2003, vol. 21, pp. 189-203.

[4] G. Bianchi, "Performance analysis of the IEEE 802.11 distributed coordination function," in IEEE Journal on Selected areas in communications, Mar. 2000, vol. 18, pp. 535-547.

[5] G. Anastasi, E. Borgia, M. Conti, and E. Gregori, "IEEE 802.11 ad hoc netowrks: performance measurements," in 23rd International distributed computing systems workshops, 2003, pp. 758-763.

[6] S. Xu and T. Saadawi, "Does the IEEE 802.11 MAC protocol worl well in multihop wireless ad hoc networks?" in IEEE Communications Magazine, Jun. 2001, pp. 130-137. 
TABLE I

RESULTS FOR THE TWO-HOP ONE-TRANSMISSION

\begin{tabular}{|l|c|c|}
\hline & original MAC & modified MAC \\
\hline packet loss, bit error & $18 \%, 0 \%$ & $10 \%, 6 \%$ \\
\hline speech w/t concealment & $1 / 10$ & $9 / 10$ \\
\hline speech w/ concealment & $0 / 10$ & $10 / 10$ \\
\hline
\end{tabular}

[7] M. I. Kazantzidis, L. Wang, and M. Gerla, "On fairness and efficiency of adaptive audio application layers for multihop wireless networks," in IEEE International Workshop on Mobile Multimedia Communications, Nov. 1999, pp. 357-362.

[8] V. N. Muthiah and W. C. Wong, "A speech-optimised multiple access scheme for a mobile ad hoc network," in First Annual Workshop on Mobile and Ad Hoc Networking and Computing, Aug. 2000, pp. 127128.

[9] H. Wu, C. Hung, M. Gerla, and R. Bagrodia, "Speech support in wireless, multihop networks," in Third International Symposium on Paprallel Architectures, Algorithms, and Networks Proceedings, Dec. 1997, pp. 282-288.

[10] I. Joe and S. G. Batsell, "Reservation CSMA/CA for multimedia traffic over mobile ad hoc networks," in IEEE International Conference on Communication, 2000, vol. 3, pp. 1714-1718.

[11] C.-H.R. Lin and M. Gerla, "A distributed control scheme in multihop packet radio networks for voice/data traffic support," in IEEE International Conference on Communication, 1995, vol. 2, pp. 12381242.

[12] C. Hoene, I. Carreras, and A. Wolisz, "Voice over IP: improving the quality over wireless LAN by adopting a booster mechanism-an experimental approach," in ITCOM, 2001.

[13] I. D. Chakeres and E. Belding-Royer, "Allowing errors in speech over wireless LANs," to be published.

[14] GSM, GSM 06.71: Digital cellular telecommuications system: Adaptive multi-rate speech processing functions; General Description, 1998.

[15] UCB/LBNL/VINT, Network Simulator- ns2, URL: http://www.isi.edu/nsnam/ns, 1997.

[16] C. E. Perkins and P. Bhagwat, "Highly dynamic destination-sequenced distance vector (DTDV) for mobile computers," Proc. of the SIGCOMM Conference on Communications Architectures, Protocols and Applications, vol. 39, pp. 234-244, 1994.

[17] E. N. Gilbert, "Capacity of a burst-noise channel," Bell Systems Technical Journal, vol. 39, pp. 1253-1265, 1960.

[18] E. O. Elliot, "Estimates of errors rates for codes on burst-noise channels," Bell Systems Technical Journal, vol. 42, pp. 1977-1997, 1963.

[19] John J. Lemmon, "Wireless link statistical bit error model," NTIA Report 02-394, U.S. Department of Commerce, June 2002. 